Journal for

... ImmunoTherapy of Cancer

\title{
among patients with COVID-19 treated with interleukin 6 (IL-6) receptor antagonist sarilumab at a single institution in Italy
}

Vincenzo Montesarchio, ${ }^{1}$ Roberto Parrella, ${ }^{2}$ Chiara lommelli, ${ }^{1}$ Antonella Bianco, ${ }^{1}$ Elio Manzillo, ${ }^{2}$ Fiorentino Fraganza, ${ }^{3}$ Cristiana Palumbo, ${ }^{4}$ Gaetano Rea, ${ }^{4}$ Patrizia Murino, ${ }^{3}$ Rosanna De Rosa (D) , ${ }^{3}$ Luigi Atripaldi, ${ }^{4}$ Maurizio D'Abbraccio, ${ }^{2}$ Marcello Curvietto, ${ }^{5}$ Domenico Mallardo, ${ }^{5}$ Egidio Celentano, ${ }^{6}$ Antonio Maria Grimaldi, ${ }^{5}$ Marco Palla, ${ }^{5}$ Claudia Trojaniello, ${ }^{5}$ Maria Grazia Vitale, ${ }^{5}$ Samuel Lewis Million-Weaver (1) , ${ }^{7}$ Paolo Antonio Ascierto (i) ${ }^{8}$

To cite: Montesarchio V, Parrella R, lommelli C, et al. Outcomes and biomarker analyses among patients with COVID-19 treated with interleukin 6 (IL-6) receptor antagonist sarilumab at a single institution in Italy. Journal for ImmunoTherapy of Cancer 2020;8:e001089. doi:10.1136/ jitc-2020-001089

- Additional material is published online only. To view please visit the journal online (http://dx.doi.org/10.1136/jitc2020-001089).

VM and RP contributed equally.

VM and RP are joint first authors.

Accepted 17 July 2020

Check for updates

(c) Author(s) (or their employer(s)) 2020. Re-use permitted under CC BY-NC. No commercial re-use. See rights and permissions. Published by BMJ.

For numbered affiliations see end of article.

Correspondence to Dr Paolo Antonio Ascierto; paolo.ascierto@gmail.com

\section{ABSTRACT}

Background The inflammatory pathology observed in severe COVID-19 disease caused by the 2019 novel severe acute respiratory syndrome coronavirus 2 (SARSCoV-2) is characterized by elevated serum levels of $\mathrm{C}$ reactive protein (CRP) and cytokines, including interferon gamma, interleukin 8 (IL-8), and interleukin 6 (IL-6). Initial reports from the outbreak in Italy, China and the USA have provided anecdotal evidence of improved outcomes with the administration of anti-IL-6 agents, and large-scale trials evaluating these therapies are ongoing.

Study description In this retrospective case series, clinical outcomes and correlates of response to treatment with the IL-6 receptor antagonist sarilumab are described for 15 patients with COVID-19 from a single institution in Southern Italy. Among 10 patients whose symptoms improved after sarilumab treatment, rapid decreases in CRP levels corresponded with clinical improvement. Lower levels of IL-6 at baseline as well as lower neutrophil to lymphocyte ratio as compared with patients whose COVID-19 did not improve with treatment were associated with sarilumab-responsive disease.

Conclusions This observation may reflect a possible clinical benefit regarding early intervention with IL-6modulatory therapies for COVID-19 and that CRP could be a potential biomarker of response to treatment.

\section{INTRODUCTION}

In December 2019, the novel severe acute respiratory syndrome coronavirus 2 (SARS-CoV-2) was identified in Wuhan, China and subsequently spread across the globe to infect as many as four million people worldwide within 5 months. ${ }^{1}$ The WHO declared the outbreak a pandemic on March 11, 2020, ${ }^{2}$ and numerous countries imposed stringent social and physical distancing orders in an effort to slow the spread. Despite these measures, the pandemic overwhelmed the capacity of healthcare systems in many areas around the world, with the northern region of Italy experiencing one of the highest mortality rates during the initial months of the crisis. ${ }^{3}$

Severe COVID-19 is associated with an acute respiratory distress syndrome (ARDS), with observations of interstitial mononuclear inflammatory infiltrates, diffuse alveolar damage, hyaline membrane formation and pulmonary edema in the lungs. ${ }^{4-6}$ Lung pathology is accompanied by pronounced inflammatory response characterized by very high levels of several cytokines in the serum, especially interleukin 6 (IL-6), IL-1 $\beta$, IL-8, interferon gamma (IFN $\gamma$ ) and tumor necrosis factor alpha. ${ }^{7-10}$ The 'cytokine storm' seen in patients with severe disease is similar to what has been described in macrophage activation syndrome (MAS)/hemophagocytic lymphohistiocytosis (HLH) ${ }^{11}{ }^{12}$ or in cytokine release syndrome (CRS) secondary to chimeric antigen receptor (CAR) $\mathrm{T}$ cell therapy. ${ }^{13}$ Administration of IL-6 blocking agents such as tocilizumab and siltuximab has been shown to be effective in reversing CAR T cell therapy-associated CRS, ${ }^{13-15}$ and tocilizumab was approved by the US Food and Drug Administration for this indication in $2017 .^{16}$ Although caution is needed in extrapolating the CAR T cell therapy experience, especially because initial data from the COVID-19 pandemic indicate that IL-6 levels are far lower in the context of SARS-CoV-2 
infection than seen in CRS, ${ }^{17} 18$ a 21-patient observational study from China reported that tocilizumab treatment could help prevent clinical deterioration of individuals with severe pneumonitis and pulmonary complications. ${ }^{19}$ Additionally, in a retrospective, observational cohort study that included 544 adult patients with severe COVID-19 pneumonia who were admitted to tertiary care centers in Bologna and Reggio Emilia, Italy, tocilizumab treatment was associated with a reduced risk of invasive mechanical ventilation or death. ${ }^{20}$

Modulation of IL-6 has emerged as a potentially promising option for COVID-19-related ARDS. ${ }^{21}$ IL-6 is a pleiotropic cytokine with nearly ubiquitous expression in stromal and immune cells. Signaling through the IL-6 receptor requires assembly on the cell membrane of a trimeric complex consisting of the cytokine bound to both its 80-kilodalton type 1 a-receptor subunit (IL$6 \mathrm{R}$, also called CD126) and a 130-kilodalton signaltransducing b-receptor glycoprotein (gp130, also called CD130). ${ }^{22}{ }^{23}$ The IL-6R exists as both a membrane-bound and soluble form, which can complex with IL-6 to bind gp130, activating the downstream signaling cascade. ${ }^{22} 23$ Several therapeutics have been developed targeting the IL-6 signaling axis, including tocilizumab and sarilumab, both of which are monoclonal antibodies that antagonize both membrane-bound and soluble IL-6R. ${ }^{24}$

At the time of manuscript preparation, several largescale trials evaluating the efficacy of IL-6-modulatory therapies have been initiated, including the international randomized, double-blind, placebo-controlled phase III COVACTA trial for tocilizumab plus standard of care in hospitalized adult patients with severe COVID-19 pneumonia, as well as the Italian TOCIVID-19 (Tocilizumab in COVID-19 Pneumonia) study, an independent phase II trial for tocilizumab in severe cases of COVID-19, which reached accrual of 330 patients within 24 hours of its announcement on March 19, 2020. A global phase II/ III trial evaluating sarilumab in hospitalized patients with severe or critical respiratory illness caused by COVID-19 was announced on March 16, 2020. ${ }^{25}$ Based on the results from the phase II portion of the study, which compared high-dose $(400 \mathrm{mg})$ versus low-dose $(200 \mathrm{mg})$ sarilumab against placebo, the trial protocol was amended so that only critical patients continued to be enrolled to receive sarilumab $400 \mathrm{mg}$ or placebo. ${ }^{26}$

As ongoing trials of IL-6-modulation in COVID-19 continue to accumulate and report data, it will be important to identify biomarkers of response to treatment as well as determine what populations of patients may derive maximal clinical benefit. In this case series, we report on clinical outcomes and hematological and inflammatory parameters in a single-arm, non-randomized retrospective analysis of 15 patients with COVID-19 from a single hospital in Italy who were treated with sarilumab. Reported here are the final results of the study. We find that all patients presented with serum IL-6 levels at least 10 times the upper limit of normal, and higher levels of IL-6 at baseline are associated with poor clinical outcomes after sarilumab administration. Furthermore, we show that decreases in $\mathrm{C}$ reactive protein (CRP) levels correlate with response to treatment. Based on these findings, early intervention with IL-6 blocking agents should be considered to prevent patients with COVID-19 from progressing to requiring intensive care support.

\section{METHODS}

\section{Enrollment and informed consent}

We report data from a retrospective case series of 15 consecutive patients (12 men, median age 59 (range 53-75)) diagnosed with SARS-COV-2 infection and treated with off-label sarilumab from March 26, 2020 to April 3, 2020 at 'Azienda Ospedaliera dei Colli - Cotugno Hospital', Italy.Informed consent for participation in the study was signed by the patients. In cases where the subject was incapable of giving informed consent and an authorized representative was not available without a delay that would, in the opinion of the investigator, compromise the potential life-saving effect of the treatment, sarilumab was administered without consent. Informed consent was obtained for use of clinical data, rendered anonymous, for purposes of clinical research, epidemiology, and study of diseases. All patients were tested for HIV, hepatitis B virus, and hepatitis $\mathrm{C}$ virus prior to treatment and were found to be negative.

Enrollment criteria were based on the Italian TOCIVID-19 study, which required patients to be hospitalized due to pneumonia with virological diagnosis of SARS-CoV-2 infection by real-time PCR and oxygen saturation at rest in ambient air $\leq 93 \%$ or requiring oxygen therapy or mechanical ventilation (either non-invasive or invasive (intubated)).

\section{Sarilumab dosing}

Sarilumab was administered as a subcutaneous injection of $400 \mathrm{mg}$. Of the 15 patients treated, 12 received a single injection of sarilumab. Similar to the protocol used in the TOCIVID-19 study, repeat dosing could be permitted if the treating physician considered that there was no improvement after the first administration, and three patients were administered a second dose within 24 hours of the first.

\section{Imaging}

Volumetric chest high recolution computed tomography (HRCT) examinations were obtained with both a 128-MDCT Ingenuity scanner (Philips Healthcare) and a 64-MDCT scanner LightSpeed VCT (General Electric Medical System, Milwaukee, Wisconsin), with patients in supine position, only when feasible, at full inspiration. Scanning parameters were $120 \mathrm{kV}$ and $100-200$ mAs, pitch 0.75-1.5, and collimation (thickness) 0.625$1.25 \mathrm{~mm}$, applying the smallest field of view according to the patient's body habitus and with adaptive filtering. Matrix size was $512 \times 512$ pixels; images were reconstructed with a $1 / 1.25 \mathrm{~mm}$ slice thickness using high 
sharp reconstruction algorithm: bone filters (HR filter). In a soft tissue filter was used to better highlight mediastinal space and any other incidental findings (nodules, pleural/pericardial effusion, lymph nodes, aortic/coronary calcifications or other findings). The whole chest volume was processed and stored on a picture archiving and communication system for retrieving images for postprocessing evaluation (PACS-OsiriX). Lung parenchyma was analyzed with a window width of 1.600 Hounsfield units (HU) and at the level of $-600 \mathrm{HU}$.

\section{Sample collection and biomarker measurement}

Blood samples were collected on days $0,1,2,3,5,7,10$, 11,12 , and 15. Blood counts and CRP were quantified as per institutional protocols. IL-6 was quantified using the Atellica Siemens platform as described previously.

\section{Anthropometric measurements}

Weight and height were obtained from patients' medical records at the time of sarilumab initiation. Body mass index (BMI) was calculated using the formula weight/ height $^{2}$ (kilograms per square meter) and classified according to the WHO categories: underweight, BMI
$<18.5$; normal, $18.5 \leq \mathrm{BMI} \leq 24.9$; overweight, $25 \leq \mathrm{BMI} \leq 29.9$; and obese, BMI $\geq 30$.

\section{RESULTS}

\section{Patient characteristics}

A total of 15 patients hospitalized for respiratory insufficiency and PCR-confirmed SARS-CoV-2 infections were treated with sarilumab as a single $400 \mathrm{mg}$ subcutaneous injection, with $3(20 \%)$ patients receiving a second dose. The median time from symptom onset to treatment was 11 days (range 6-21). Dates of symptom onset, hospitalization, sarilumab administration and arterial oxygen pressure to fractional inspired oxygen $\left(\mathrm{PaO}_{2}: \mathrm{FiO}_{2}\right)$ values before and after treatment for all patients are shown in table 1. The median $\mathrm{PaO}_{2}: \mathrm{FiO}_{2}$ at baseline for all patients was 122 (range 83-240), and 8 of 15 (53.3\%) were intubated at the time of sarilumab administration.

The median age of all treated patients was 59 (range 53-75). Consistent with emerging evidence that men are more likely to develop serious disease, ${ }^{2728} 80 \%$ (12) of patients in the cohort were men and $20 \%$ (3) were

Table 1 Respiratory parameters for all patients before and after sarilumab administration

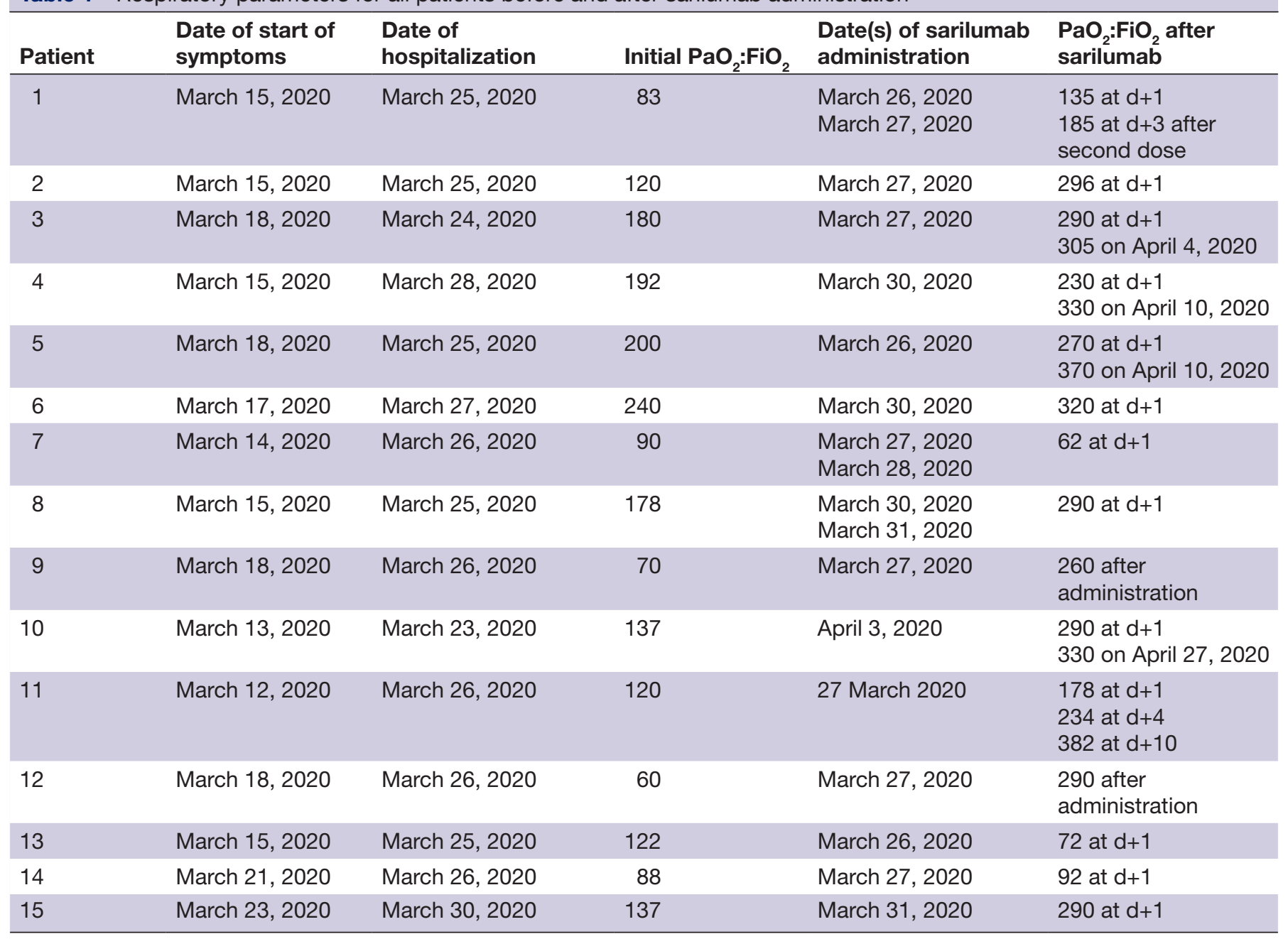

$\mathrm{FiO}_{2}$, fractional inspired oxygen; $\mathrm{PaO}_{2}$, arterial oxygen pressure. 
women. Of the 10 patients for whom BMI values were available, 2 were of normal weight $(18.5<$ BMI $\leq 24.9), 5$ were overweight $(25<\mathrm{BMI} \leq 29.9)$ and 3 were obese (BMI $>30$ ), in line with reports that overweight/obesity may be a risk factor for severe respiratory complications in COVID19. ${ }^{29} 30$ Comorbidities were present in five patients, including two with hypertension, two with diabetes, one with chronic obstructive pulmonary disease and dilatative cardiomyopathy, and one with opioid addiction. All patients received hydroxychloroquine $200 \mathrm{mg}$ two times per day, lopinavir/ritonavir $800 \mathrm{mg} / 800 \mathrm{mg}$ two times per day, and heparin 4000-6000 IU two times per day. Eleven patients were also treated with methylprednisolone at $80 \mathrm{mg} /$ day for $5-7$ days.

\section{Safety}

No injection reactions or dermatological toxicities were reported. Liver toxicity was not detected. No patients reported gastrointestinal or metabolic adverse events. One patient developed grade 2 thrombocytopenia according to the 2020 version of the Common Terminology Criteria for Adverse Events (V.5.0). This patient had established heparin treatment prior to sarilumab administration at the time of thrombocytopenia onset. However it cannot be ruled out as a side effect of sarilumab.

\section{Outcomes}

Rapid improvements in respiratory parameters were observed in $10(67 \%)$ patients after sarilumab administration, enabling their eventual hospital discharge or transfer to subintensive care. Three patients required two doses of sarilumab due to an investigator-assessed lack of improvement after the first administration, and all displayed increased $\mathrm{PaO}_{2}: \mathrm{FiO}_{2}$ after receiving the second dose. A total of five patients who received sarilumab died. All patients who died were intubated and on ventilators before sarilumab treatment. Among the eight patients in total who required mechanical ventilation prior to sarilumab administration, three displayed sufficient improvement in pulmonary function after treatment to allow for extubation, although one died 5 days after being extubated due to a massive embolism.

The median baseline $\mathrm{PaO}_{2}: \mathrm{FiO}_{2}$ among patients with disease that responded to sarilumab was 121, and among patients who died the median $\mathrm{PaO}_{2}: \mathrm{FiO}_{2}$ was 178 . One day after sarilumab administration, the median $\mathrm{PaO}_{2}: \mathrm{FiO}_{2}$ was 270. The median $\mathrm{PaO}_{2}: \mathrm{FiO}_{2}$ value on $\mathrm{d}+1$ for patients with sarilumab-responsive disease was 280 , whereas the median value at the same time point for patients who died was 178. Respiratory parameters for all patients are summarized in table 1.

Among the 10 patients whose disease responded to sarilumab treatment, 2 were of normal weight $(18.5<\mathrm{BMI} \leq 24.9), 4$ were overweight $(25<\mathrm{BMI} \leq 29.9), 2$ were obese $(\mathrm{BMI} \geq 30)$, and $\mathrm{BMI}$ measurements were not available for 2 patients. Of the five patients who died, one was overweight $(25<\mathrm{BMI} \leq 29.9)$, two was obese $(\mathrm{BMI} \geq 30)$

\begin{tabular}{ll}
\hline Table 2 Patient demographics & \\
\hline Patient characteristics & $\mathbf{N}=\mathbf{1 5}$ \\
\hline Gender: female/male, $\mathrm{n}(\%)$ & $3(20) / 12(80)$ \\
\hline Median age & $59($ range 53-75) \\
\hline Median $\mathrm{PaO}_{2}: \mathrm{FiO}_{2}$ & $122(83-240)$ \\
\hline Median $\mathrm{BMl}$ & 28.7 (range 23-45) \\
\hline Normal weight (18.5<BMl $\leq 24.9), \mathrm{n}(\%)$ & $2(13.3)$ \\
\hline Overweight $(25<\mathrm{BMI} \leq 29.9), \mathrm{n}(\%)$ & $5(33.3)$ \\
\hline Obese (BMI $\geq 30), \mathrm{n}(\%)$ & $3(20)$ \\
\hline NA, $\mathrm{n}(\%)$ & $5(33.3)$ \\
Intubated, $\mathrm{n}(\%)$ & $8(53.3)$ \\
\hline Not intubated, $\mathrm{n}(\%)$ & $7(46.7)$ \\
\hline Deaths, $\mathrm{n}(\%)$ & $5(33.3)$ \\
\hline
\end{tabular}

$\mathrm{BMI}$, body mass index; $\mathrm{FiO}_{2}$, fractional inspired oxygen; $\mathrm{PaO}_{2}$, arterial oxygen pressure.

and BMI measurements were not available for 3 patients (table 2).

\section{Radiological findings}

Almost $75 \%$ of patients with COVID-19 ARDS present with abnormal findings on chest CT scans, most commonly ground-glass opacities, which are reported in more than half of patients. Other commonly reported findings include patchy infiltrates and interstitial lung disease, although minimal imaging abnormalities are seen in some patients. ${ }^{8103132}$ In the treated cohort, abnormal CT imaging features were observed in the lungs of all patients, including ground-glass opacities and crazy paving patterns, which have been described as characteristics of COVID-19. ${ }^{32}{ }^{33}$ Pre-sarilumab and post-sarilumab CT scans were available for 11 patients, 9 of whom had disease that responded to treatment while 2 did not. Radiographic improvement was observed in all patients who responded to treatment. Patients repeated a highresolution chest CT scan after 4 and 8 weeks. Radiological response was assessed by reducing lung consolidation areas and improvement of parenchymal ground-glass. Representative CT scans from one patient whose disease responded to sarilumab and from one patient who died are depicted in figure 1. Radiographs from all patients for whom imaging was available can be found in online supplementary figure 1 .

\section{Baseline hematological and inflammatory values predictive of responsive disease}

Clinical improvement after sarilumab administration was associated with lower baseline values of several hematological and inflammatory parameters. Baseline values for all patients as well as grouped by response to treatment are summarized in table 3. Small differences in baseline d-dimer levels were noted, with a higher median value in patients who died compared with those with responsive disease $(1884 \mathrm{ng} / \mathrm{mL}$, range $464-4250$ vs $1127 \mathrm{ng} /$ 


\section{a. Patient with responsive disease}

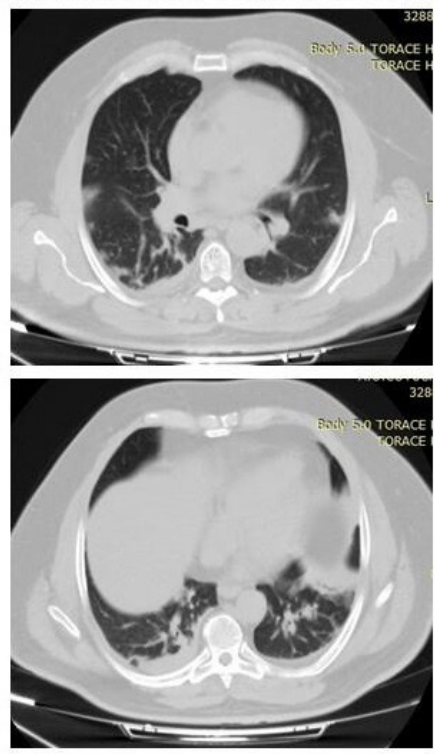

Pre-treatment
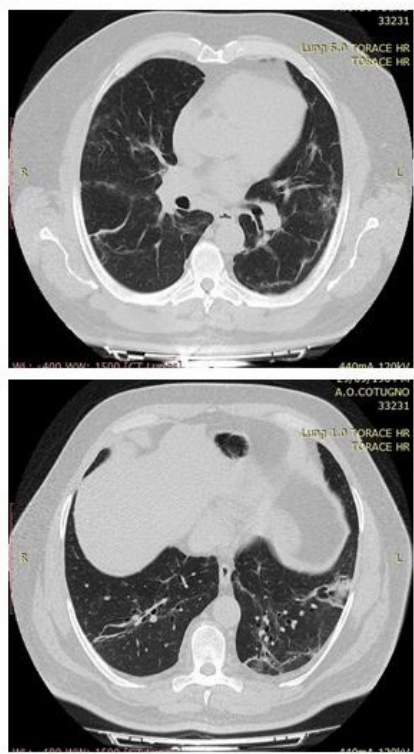

Day +12

\section{b. Patient with non-responsive disease}
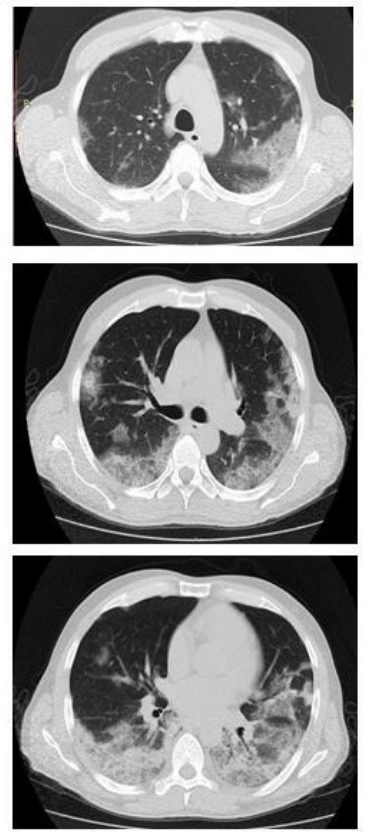

Pre-treatment
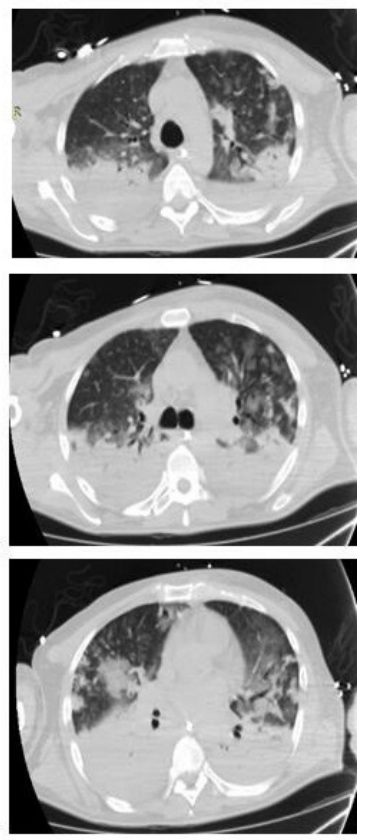

Day +9

Figure 1 H Representative chest CT scans pre- and post-treatment from patients with sarilumab responsive(a) and nonresponsive (b) COVID-19.

mL, range 200-2907). The median baseline platelet counts were initially slightly higher in patients who died compared with those whose disease responded to sarilumab $\left(320 \times 10^{9} / \mu \mathrm{L}\right.$, range $138-535$ vs $291 \times 10^{9} / \mu \mathrm{L}$, range 127-393); however, a rapid-onset thrombocytopenia associated with clinical decline was subsequently observed.

In line with published observations of neutrophil to lymphocyte ratio (NLR) as a prognostic factor for poor outcomes in COVID-19, ${ }^{34} 35$ the median value among patients who died was roughly twofold higher than in those whose symptoms improved after sarilumab (median NLR=19.5, range 14.6-25.2 vs median NLR=9.5, range 1.9-22.7). Dramatic differences in baseline CRP levels were also observed between the two groups, with a median value of $12.9 \mathrm{mg} / \mathrm{dL}$ (range 3.5-27.8) among 10 patients whose disease responded to treatment compared with 29.5 (range 1.1-30.5) in 5 patients who died.

Elevated IL-6 levels were observed in all patients, with a median value of $61.9 \mathrm{pg} / \mathrm{mL}$ (range 5.2-1000). The measured values were all higher than those seen under normal homeostatic conditions, where serum concentrations of IL-6 are typically lower than picograms per milliliter, but lower than the levels seen in severe CRS, which can reach nanograms per milliliter, ${ }^{17}$ or fatal sepsis, where up to micrograms of IL-6 per milliliter have been reported. ${ }^{36}$ Notably, IL-6 levels at baseline were lower overall in patients who displayed clinical improvement after sarilumab. Among the group whose disease responded to sarilumab, the median baseline IL-6 was $56.6 \mathrm{pg} / \mathrm{mL}$ (range 5.2-81.5) compared with $213 \mathrm{pg} /$ $\mathrm{mL}$ (range 60.5-1000) in those who died. The differences observed between the two groups suggest that the timing of intervention with sarilumab during the clinical course of COVID-19 inflammatory pathology may be an important determinant of efficacy.

\section{Biomarkers of response to treatment}

Hematological and inflammatory parameters were monitored for 15 days after sarilumab administration. Consistent with published observations, ${ }^{34} 35$ 37-39 all patients presented with elevated IL-6, CRP and d-dimer combined with high NLR and thrombocytopenia, signatures that have been described as indicative of disseminated intravascular coagulation and/or cytokine storm. ${ }^{3840}$ At baseline, elevated NLR was observed in all patients (median 14.6, range $1.8-25.1$ ), with values $\geq 10$ measured in 10 patients $(66.6 \%)$. All patients for whom measurements were available displayed elevated levels of CRP (median $15 \mathrm{mg}$ / $\mathrm{dL}$, range 1.1-30.5) as well as IL-6 (median $61.9 \mathrm{pg} / \mathrm{mL}$, range $5.2-1000 \mathrm{pg} / \mathrm{mL}$ ). Pronounced thrombocytopenia was present at baseline in $14(93.3 \%)$ patients, and the median platelet count was $306 \times 10^{9} / \mu \mathrm{L}$ (range $127-535$ ). In patients who died, platelet counts decreased dramatically in 2 days after sarilumab treatment, whereas levels remained relatively constant throughout the course of the study for patients who experienced clinical improvement. Among the 11 patients for whom d-dimer measurements were available before treatment, levels were greater than two times the upper normal limit in eight patients $(53.3 \%)$. The median d-dimer value for the entire cohort was $1151 \mathrm{ng} / \mathrm{mL}$ (range $200-4250$ ).

In all patients, a decrease in white cell counts and absolute neutrophil counts was observed after sarilumab administration. Reductions in d-dimer after sarilumab 
Table 3 Median baseline hematological and inflammatory values

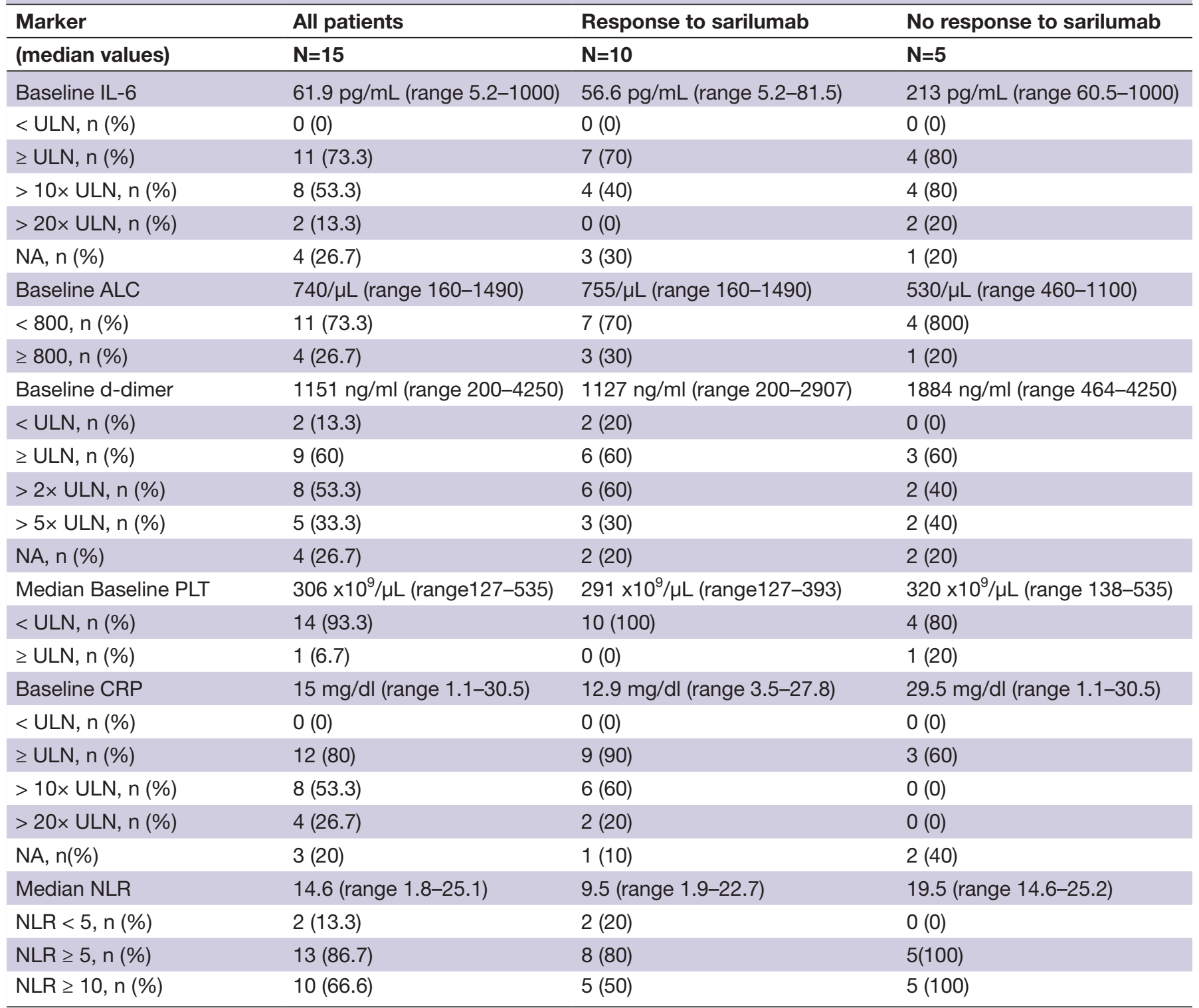

ALC, Absolute Lymphocte Count; CRP, C reactive protein; IL-6, interleukin 6; NA, Not Available; NLR, neutrophil to lymphocyte ratio; PLT, platelet; ULN, upper limit of normal.

were also observed across all patients (online supplementary figure 2). Distinct patterns in the values for several markers did emerge between patients whose disease responded and those who died, most notably for CRP, IL-6, NLR, and eosinophil counts. The average values and box plots for IL-6, CRP, NLR, eosinophils and platelets are presented in figure 2.

Among patients with COVID-19 that improved after treatment, dramatic reductions in serum CRP were observed immediately following sarilumab administration, with levels mostly decreasing to below the upper normal limit within 5 days. Although serum CRP did decrease after sarilumab in patients who died, levels among these patients never declined below the highest values seen in the group with responding disease (figure 2A). The reduction in CRP levels among responding patients corresponded with improvements in respiratory parameters. Notably, serum IL-6 levels increased after sarilumab in both patients whose disease responded to treatment and those who died, a phenomenon that has also been observed in patients receiving the IL-6R antagonist tocilizumab for CRS subsequent to CAR T cell therapy. ${ }^{41}$ Among the patients whose symptoms improved after treatment, the increase was transient, persisting for no longer than 2 days, with a sharp subsequent decrease and IL-6 levels reaching a steady low point from day +9 onward. By contrast, IL-6 levels continuously increased among patients who died, eventually reaching values almost 100 times the normal ranges (figure 2B). In this cohort, CRP values were more informative than IL-6 measurements as a surrogate for response to treatment. 
a.

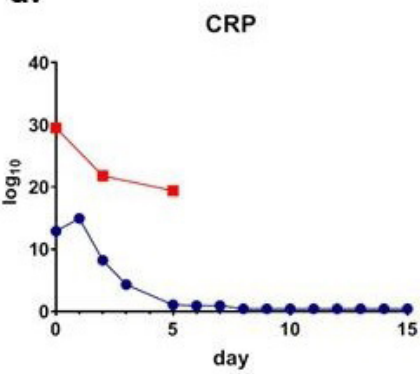

d.

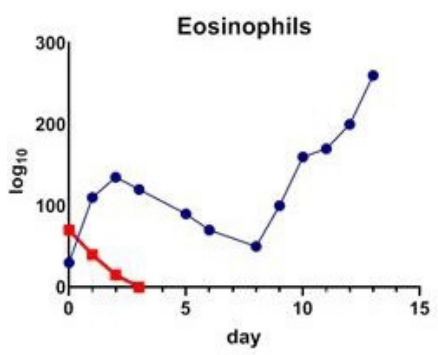

b.

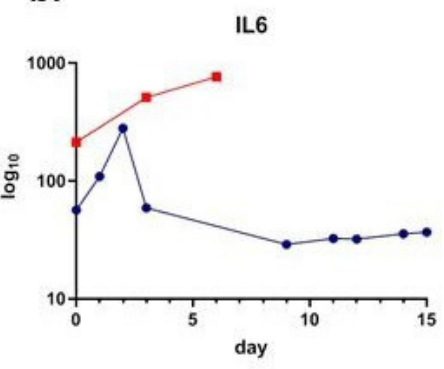

e.
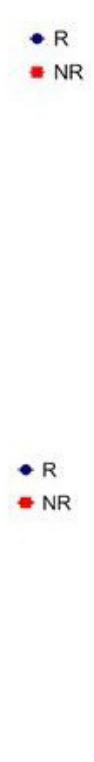

- $\mathrm{R}$

NR

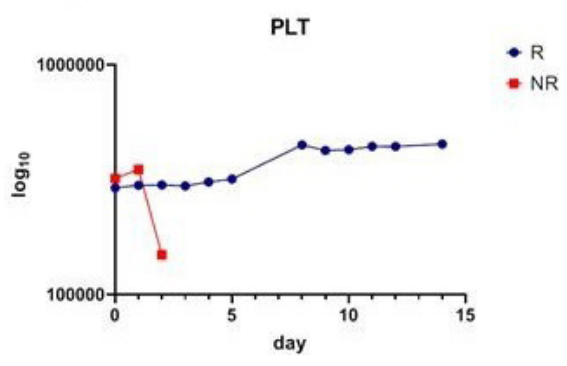

c.

- R

- NR

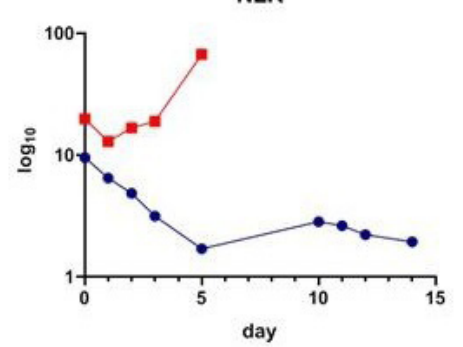

- R

- NR

Figure 2 Hematologicand inflammatory parameters in patients with sarilumab responsive (bluemarkers) and non-responsive (red markers) COVID-19. Mean values for c-reactiveprotein (CRP) (panel a), interleukin 6 (IL-6) (panel b),neutrophil-tolymphocyte ratio (NLR) (panel c), eosinophils (panel d) andplatelets (panel e) at baseline (day 0) as well as days 1, 2, 3, 5, 7, 10, 11,12 , and 15 after sarilumab administration are shown.

NLR steadily decreased over 5 days after administration in patients whose disease responded to sarilumab, whereas average values increased continually among patients who died (figure 2C). Similar patterns were observed with the derived NLR (online supplementary figure 2). Total white cell counts slightly decreased with similar kinetics for the first 3 days after sarilumab administration in both groups; however, a modest rebound was observed between days +5 and +15 in patients whose disease responded to treatment. Absolute lymphocyte counts remained relatively stable for 15 days after sarilumab administration among patients with responding disease, with a concomitant steep decrease in absolute neutrophil counts during the first 5 days. In patients who died, lymphocyte counts decreased steadily and only modest reductions in absolute neutrophil counts were observed during the first 3 days after sarilumab treatment.

Although the role that eosinophils play in response to SARS-CoV-2 infection has yet to be definitively characterized, a retrospective review of 85 fatal cases of COVID-19 in Wuhan, China found that $81 \%$ of patients were eosinopenic at the time of hospital admission. ${ }^{42}$ In sarilumabtreated cohort, eosinophilia was associated with improved outcomes after administration (figure 2D). A marked eosinophilia was observed in patients whose disease improved after sarilumab, with sharp escalation in eosinophil counts in the first 2 days after administration followed by a transient decrease between days +3 and +8 and then a subsequent increase throughout the end of the observation period. By contrast, eosinophil counts declined steadily in patients who died.

\section{DISCUSSION}

This case series describes 15 patients with COVID-19 who were treated with sarilumab. Rapid improvements in respiratory function as well as normalization of inflammatory markers were observed in 10 of 15 patients. Preliminary analysis of hematological and inflammatory parameters indicates that elevated values of CRP, IL-6 and the NLR were associated with worse outcomes and a lack of response to treatment. Although these results will need to be validated in larger patient cohorts and randomized trials, our study indicates that a subset of patients with COVID-19 may benefit from treatment with sarilumab or other IL-6-modulatory therapies.

Limitations of this study include the relatively small number of patients treated and the lack of randomization. In this study, 8 of $15(53 \%)$ patients were intubated at the time of sarilumab administration, and the mortality rate among patients requiring mechanical ventilation was $62.5 \%$. The proportion of intubated patients in this cohort is lower than the $88 \%$ rate published in a retrospective analysis of 1591 patients with confirmed SARS-CoV-2 infections who were admitted to ICUs in Lombardy, Italy between February 20 and March 18, 2020. ${ }^{43}$ The rates in this cohort as well as in Italy overall are higher than those reported in other countries, as illustrated by an analysis of 5700 patients in New York City, where 20.2\% (1151) required mechanical ventilation and of these $24.5 \%$ (282) died. ${ }^{44}$ Several factors have been proposed to account for the disproportionately high fatality rate observed thus far in Italy compared with other countries, including the older age of the population and testing and case reporting disparities. 
Comedication with other COVID-19 therapies is another potential confounding factor, as all patients in this cohort received hydroxychloroquine, lopinavir/ ritonavir, and heparin. Additionally, 11 patients were also treated with methylprednisolone. Hydroxychloroquine likely had minimal effects on the results. The initial study purporting improved outcomes with hydroxychloroquine for the treatment of COVID-19 was retracted, ${ }^{45}$ and subsequent analysis from the UK's RECOVERY trial found no benefit for the drug in hospitalized patients when compared with standard of care. ${ }^{46}$ Similarly, a randomized, controlled, open-label trial of lopanivir/ritonavir for hospitalized adult patients with confirmed SARS-CoV-2 infection found no differences in time to clinical improvement with the antiviral compared with standard of care and overall similar mortality rates at 28 days $(19.2 \%$ vs $25.0 \%$; difference, -5.8 percentage points; $95 \%$ CI -17.3 to 5.7). ${ }^{47}$ Steroid use has reported more encouraging results for COVID-19. In the RECOVERY trial, which compared outcomes for 2104 patients randomly allocated to receive dexamethasone with 4321 patients concurrently allocated to usual care, dexamethasone reduced deaths by roughly one-third in patients receiving invasive mechanical ventilation $(29.0 \%$ vs $40.7 \%$, rate ratio (RR) 0.65 (95\% CI 0.51 to 0.82$) ; \mathrm{p}<0.001$ ) and by approximately one-fifth in patients receiving oxygen without invasive mechanical ventilation (21.5\% vs $25.0 \%$, RR 0.80 (95\% CI 0.70 to 0.92$) ; \mathrm{p}=0.002)$, although it did not reduce mortality in patients not receiving respiratory support at randomization ( $17.0 \%$ vs $13.2 \%$, RR 1.22 (95\% CI 0.93 to 1.61$)$; $\mathrm{p}=0.14) .{ }^{48}$ Methylprednisolone has also been reported to reduce risk for mechanical ventilation. In a case series of 15 severe and critical patients with COVID-19 in China who received pulse single dosage of $40-500 \mathrm{mg}$ methylprednisolone, oxygenation improved significantly, no deaths occurred and only one needed mechanical ventilation. $^{49}$

Although caution must be exercised when comparing across trials, the outcomes observed in this case series roughly correspond to the topline results that were announced for the global randomized phase II/III trial of sarilumab for critically ill patients with COVID-19. In the trial, of the 88 patients in the critical group who received high-dose sarilumab (400 mg), $52(59 \%)$ achieved clinical improvement after treatment and $20(23 \%)$ died. ${ }^{26}$

Modulation of the severe inflammatory state associated with severe COVID-19 may offer a potentially important strategy to limit pulmonary complications of the disease, reducing the necessity for intensive care unit support and ultimately mortality. It is evident, however, that the clinical course of SARS-CoV-2 infection is highly heterogeneous, with presentations ranging from mild respiratory symptoms to irreversible respiratory failure. ${ }^{432} 50$ Furthermore, the ultimate etiology of acute respiratory distress in COVID-19 remains a matter of some controversy, with the relative contributions of unrestrained viral replication versus hyperinflammation still incompletely understood. ${ }^{5152}$ The timing of intervention may be important, as treatment with anti-IL-6 therapies too early in the course of infection could hinder the eventual development of a protective adaptive immune response. ${ }^{53}$ Conversely, the benefit of IL-6 modulation may be limited in cases where the hyperinflammatory cascade is too far advanced. ${ }^{545}$ Multiple strategies may be needed due to the overlapping regulation of proinflammatory cytokines. Ongoing trials are investigating agents directed against granulocytemacrophage colony-stimulating factor (GM-CSF), IFN $\gamma$ and IL-1, among others. ${ }^{24}$ Encouraging results have been reported in a small-scale study of the IL-1 receptor antagonist anakinra in hospitalized patients with COVID19 , where a reduced risk of invasive mechanical ventilation or death was observed with treatment compared with historical controls (HR 0.22, 95\% CI 0.11 to 0.41 ; $\mathrm{p}<0.0001) .{ }^{56}$ Identifying biomarkers of response to treatment with sarilumab or other IL-6-modulatory therapies will be important for the ultimate incorporation of these strategies into widespread use. Among patients studied here, the most reliable marker of clinical response was CRP, which declined steeply immediately after sarilumab administration among patients with responsive disease. Although IL-6 levels also eventually decreased, the initial kinetics (characterized by a transient increase after treatment) make measurements of the cytokine non-ideal for clinical decision making. Furthermore, given that same-day access to IL-6 levels may be limited in some hospitals, CRP is a more useful marker of real-time evaluation of the effectiveness of treatment.

One striking difference between sarilumab-responsive and non-responsive disease was the dramatic eosinopenia observed in patients who died after treatment and the biphasic pattern of eosinophilia seen in patients who recovered. Eosinophils are protective against respiratory tract infections, although they also play a role in inflammatory airway pathology such as asthma. ${ }^{57}$ Eosinophils have been conspicuously absent from lung samples in published analyses from autopsies both early in the disease and postmortem, ${ }^{58}$ and the characteristic pattern of immune infiltration associated with infection is typically dominated by monocytes and mononuclear cells. ${ }^{59}$ Although type 1 immunity is likely important early on in the course of infection, dysregulated inflammatory responses may contribute to pathology, and severe disease has been associated with a characteristic proinflammatory T helper 17 signature for both SARS-CoV-2 infections and the Middle East respiratory syndrome (MERS) coronavirus. ${ }^{60}$ Given the importance of type 2 immunity for both the generation of high antibody titers and resolution of inflammation, ${ }^{62}$ the eosinophilia observed after sarilumab administration in patients who recovered may represent an important hallmark of infection control.

Elevated levels of CRP, IL-6, and NLR have previously been described as predictive of poor outcomes in COVID19. ${ }^{4183435}$ In the cohort described in this paper, response to sarilumab was associated with lower values at baseline for these markers. Experience from the use of the IL-6R antagonist tocilizumab in China ${ }^{19}$ has indicated that early 
administration of cytokine-modulatory therapy is critical, ideally before symptoms start to rapidly deteriorate at the onset of the cytokine storm. ${ }^{63}$ Taken with the results of this case series, the optimal time for intervention with anti-IL-6 agents may be early in the disease course. Patients with oxygen saturation at rest and without supply $\leq 93 \%$ should be closely monitored and anti-IL-6 should be strongly considered at the first signs of increases in CRP.

Although the SARS-CoV-2 outbreak appears to have peaked in some regions around the world with new cases and fatalities beginning to decline week by week, the healthcare system remains stretched thin. Supplies of personal protective equipment are running low in multiple areas and shortages of several crucial drugs are predicted. ${ }^{64}$ Effective interventions to reduce the number of patients requiring intensive care are still needed, especially as some areas relax physical distancing mandates and cases rebound. In all likelihood, effective management of COVID-19 will require a strategy encompassing antivirals, multiple anticytokine agents and/or passive immunization using convalescent plasma. Examples of such approaches include the ongoing REMDACTA trial of tocilizumab in combination with remdesivir. Although initial results indicating clinical benefit with the use of sarilumab and other cytokine-modulatory therapies have begun to be reported, further definitive prospective randomized trials should also be conducted with all due haste. Additionally, future studies should further characterize immune and inflammatory responses throughout the course of SARS-CoV-2 infection as well as after treatment. A collaborative and multidisciplinary approach will be needed in order to reduce the human toll of this unprecedented and devastating pandemic.

\author{
Author affiliations \\ ${ }^{1}$ Department of Medical Oncology, Azienda Ospedaliera dei Colli-Monaldi Hospital, \\ Napoli, Italy \\ ${ }^{2}$ Department of Infectious Diseases and Infectious Diseases Emergencies, Azienda \\ Ospedaliera dei Colli-Cotugno Hospital, Napoli, Italy \\ ${ }^{3}$ Critical Area Department, Azienda Ospedaliera dei Colli-Cotugno and Monaldi \\ Hospital, Napoli, Italy \\ ${ }^{4}$ Department of Health Services, Azienda 0spedaliera dei Colli-Cotugno and Monaldi \\ Hospital, Napoli, Italy \\ ${ }^{5}$ Department of Melanoma, Cancer Immunotherapy and Development Therapeutics, \\ Istituto Nazionale Tumori IRCCS Fondazione Pascale, Napoli, Campania, Italy \\ ${ }^{6}$ Epidemiology and Biostatistics Unit, Istituto Nazionale Tumori IRCCS Fondazione \\ Pascale, Napoli, Campania, Italy \\ ${ }^{7}$ Independent Investigator, Madison, Wisconsin, USA \\ ${ }^{8}$ Istituto Nazionale Tumori IRCCS Fondazione Pascale, Napoli, Italy
}

Correction notice This paper has been corrected since it was published online. The author name Roberto Parrella was incorrectly spelt as Roberto Parella.

Twitter Vincenzo Montesarchio @Vincenzo Montesarchio and Samuel Lewis Million-Weaver @millionweaver

Acknowledgements The authors thank the patients and their families. The authors also thank the management of the hospitals for the strong support in their daily activities during the COVID-19 emergency: Dr Maurizio Di Mauro, General Manager of Azienda dei Colli of Napoli, Italy; Dr Attilio Bianchi, General Manager of Istituto Nazionale Tumori IRCCS Fondazione Pascale, Napoli, Italy; and Dr Gerardo Botti, Scientific Director of Istituto Nazionale Tumori IRCCS Fondazione Pascale, Napoli, Italy.
Contributors PAA conceptualized the study and provided leadership in determining manuscript structure and content, as well as collection and interpretation of clinical data. Collected and interpreted the data, and provided key insights throughout the duration of the study. Cl, AB, EM, FF, CP, GR, PM, RDR, LA, MDA, MC, DM, EC, AMG, $\mathrm{MP}, \mathrm{CT}$, and MGV assisted with data collection and interpretation and provided valuable discussions assisting in the preparation of the paper. SMW wrote the paper and made the figures. All authors reviewed and edited the drafts of the manuscript.

Funding The authors have not declared a specific grant for this research from any funding agency in the public, commercial or not-for-profit sectors.

Competing interests AMG: advisory and consultant role for BMS, MSD and Novartis; and travel grants from BMS, Merck Serono, Pierre Fabre, Roche and Novartis. PAA: consultant/advisory role for Bristol-Myers Squibb, Roche-Genentech, Merck Sharp \& Dohme, Array, Novartis, Merck Serono, Pierre Fabre, Incyte, NewLink Genetics, Genmab, Medlmmune, AstraZeneca, Syndax, SunPharma, Sanofi, Idera, Ultimovacs, Sandoz, Immunocore, 4SC, Alkermes, Italfarmaco, and Nektar; research funds from Bristol-Myers Squibb, Roche-Genentech, and Array; and travel support from MSD.

\section{Patient consent for publication Not required.}

Ethics approval The study was conducted under the Declaration of Helsinki and was approved by the departmental review board.

Provenance and peer review Not commissioned; externally peer reviewed.

Open access This is an open access article distributed in accordance with the Creative Commons Attribution Non Commercial (CC BY-NC 4.0) license, which permits others to distribute, remix, adapt, build upon this work non-commercially, and license their derivative works on different terms, provided the original work is properly cited, appropriate credit is given, any changes made indicated, and the use is non-commercial. See http://creativecommons.org/licenses/by-nc/4.0/.

\section{ORCID iDs}

Rosanna De Rosa http://orcid.org/0000-0001-8684-0008

Samuel Lewis Million-Weaver http://orcid.org/0000-0001-9057-6326

Paolo Antonio Ascierto http://orcid.org/0000-0002-8322-475X

\section{REFERENCES}

$1 @$ OurWorldlnData. Total confirmed COVID-19 deaths: how rapidly are they increasing? : @OurWorldlnData, 2020. Available: https:// ourworldindata.org/grapher/covid-confirmed-deaths-since-5th-death

2 Fauci AS, Lane HC, Redfield RR. Covid-19 - Navigating the Uncharted. N Engl J Med Overseas Ed 2020;382:1268-9.

3 Livingston E, Bucher K. Coronavirus disease 2019 (COVID-19) in Italy. JAMA 2020;323:1335.

4 Chen G, Wu D, Guo W, et al. Clinical and immunological features of severe and moderate coronavirus disease 2019. J Clin Invest 2020;130:2620-9.

5 Tian S, Hu W, Niu L, et al. Pulmonary pathology of early-phase 2019 novel coronavirus (COVID-19) pneumonia in two patients with lung cancer. J Thorac Oncol 2020;15:700-4.

6 Xu Z, Shi L, Wang Y, et al. Pathological findings of COVID-19 associated with acute respiratory distress syndrome. Lancet Respir Med 2020;8:420-2.

7 Conti P, Ronconi G, Caraffa A, et al. Induction of pro-inflammatory cytokines (IL-1 and IL-6) and lung inflammation by Coronavirus-19 (COVI-19 or SARS-CoV-2): anti-inflammatory strategies. J Biol Regul Homeost Agents 2020;34. doi:10.23812/CONTI-E. [Epub ahead of print: 14 Mar 2020].

8 Mo $P$, Xing $Y, X i a o Y$, et al. Clinical characteristics of refractory COVID-19 pneumonia in Wuhan, China. Clin Infect Dis 2020.

9 Qin C, Zhou L, Hu Z, et al. Dysregulation of immune response in patients with COVID-19 in Wuhan, China. Clin Infect Dis 2020. doi:10.2139/ssrn.3541136. [Epub ahead of print: 12 Mar 2020].

10 Wang Z, Yang B, Li Q, et al. Clinical features of 69 cases with coronavirus disease 2019 in Wuhan, China. Clin Infect Dis 2020;367.

11 La Rosée P. Treatment of hemophagocytic lymphohistiocytosis in adults. Hematology Am Soc Hematol Educ Program 2015;2015:190-6.

12 Tamamyan GN, Kantarjian HM, Ning J, et al. MalignancyAssociated hemophagocytic lymphohistiocytosis in adults: relation to hemophagocytosis, characteristics, and outcomes. Cancer 2016;122:2857-66.

13 Brudno JN, Kochenderfer JN. Toxicities of chimeric antigen receptor T cells: recognition and management. Blood 2016;127:3321-30. 
14 Neelapu SS. Managing the toxicities of car T-cell therapy. Hematol Oncol 2019;37 Suppl 1:48-52.

15 Santomasso B, Bachier C, Westin J, et al. The other side of car T-cell therapy: cytokine release syndrome, neurologic toxicity, and financial burden. Am Soc Clin Oncol Educ Book 2019;39:433-44.

16 Kotch C, Barrett D, Teachey DT. Tocilizumab for the treatment of chimeric antigen receptor $T$ cell-induced cytokine release syndrome. Expert Rev Clin Immunol 2019;15:813-22.

17 Teachey DT, Lacey SF, Shaw PA, et al. Identification of predictive biomarkers for cytokine release syndrome after chimeric antigen receptor T-cell therapy for acute lymphoblastic leukemia. Cancer Discov 2016:6:664-79.

18 Ruan Q, Yang K, Wang W, et al. Clinical predictors of mortality due to COVID-19 based on an analysis of data of 150 patients from Wuhan, China. Intensive Care Med 2020;46:846-8.

19 Xea X. Effective treatment of severe COVID19 patients with tocilizumab. Chinaxiv. 2020.

20 Guaraldi G, Meschiari M, Cozzi-Lepri A, et al. Tocilizumab in patients with severe COVID-19: a retrospective cohort study. Lancet Rheumatol 2020.

21 Ascierto PA ea. SITC Statement on anti-IL-6/IL-6R for COVID-19 - Society for Immunotherapy of Cancer (SITC), 2020. Available: https://www.sitcancer.org/research/covid-19-resources/il-6editorial

22 Tanaka T, Narazaki M, Kishimoto T. II-6 in inflammation, immunity, and disease. Cold Spring Harb Perspect Biol 2014;6:a016295.

23 Uciechowski P, Dempke WCM. Interleukin-6: a Masterplayer in the cytokine network. Oncology 2020;98:131-7.

24 Arnaldez FI, O'Day SJ, Drake CG, et al. The Society for immunotherapy of cancer perspective on regulation of interleukin-6 signaling in COVID-19-related systemic inflammatory response. $J$ Immunother Cancer 2020;8:e000930.

25 Regeneron and Sanofi Begin Global Kevzara $\AA$ (sarilumab) Clinical Trial Program in Patients with Severe COVID-19|Regeneron Pharmaceuticals Inc. [press release] 2020.

26 Regeneron and Sanofi Provide Update on U.S. Phase 2/3 AdaptiveDesigned Trial of Kevzara ${ }^{8}$ (sarilumab) in Hospitalized COVID-19 Patients | Regeneron Pharmaceuticals Inc. [press release] 2020.

27 Jin J-M, Bai P, He W, et al. Gender differences in patients with COVID-19: focus on severity and mortality. Front Public Health 2020;8:152.

$28 \mathrm{Cai} \mathrm{H}$. Sex difference and smoking predisposition in patients with COVID-19. Lancet Respir Med 2020;8:e20-e.

29 Kalligeros M, Shehadeh F, Mylona EK, et al. Association of obesity with disease severity among patients with coronavirus disease 2019 . Obesity 2020;28:1200-1204

30 Simonnet A, Chetboun M, Poissy J, et al. High prevalence of obesity in severe acute respiratory syndrome coronavirus-2 (SARS-CoV-2) requiring invasive mechanical ventilation. Obesity 2020;28:1195-9.

31 Qian G-Q, Yang N-B, Ding F, et al. Epidemiologic and clinical characteristics of 91 hospitalized patients with COVID-19 in Zhejiang, China: a retrospective, multi-centre case series. QJM 2020;113:474-81.

32 Rodriguez-Morales AJ, Cardona-Ospina JA, Gutiérrez-Ocampo $\mathrm{E}$, et al. Clinical, laboratory and imaging features of COVID-19: a systematic review and meta-analysis. Travel Med Infect Dis 2020;101623.

33 Shi $\mathrm{H}$, Han X, Jiang N, et al. Radiological findings from 81 patients with COVID-19 pneumonia in Wuhan, China: a descriptive study. Lancet Infect Dis 2020;20:425-34.

34 Liu Y, Du X, Chen J, et al. Neutrophil-To-Lymphocyte ratio as an independent risk factor for mortality in hospitalized patients with COVID-19. J Infect 2020.

35 Yang A-P, Liu J-P, Tao W-Q, et al. The diagnostic and predictive role of NLR, d-NLR and PLR in COVID-19 patients. Int Immunopharmacol 2020;84:106504.

36 Oda S, Hirasawa $\mathrm{H}$, Shiga $\mathrm{H}$, et al. Sequential measurement of IL-6 blood levels in patients with systemic inflammatory response syndrome (SIRS)/sepsis. Cytokine 2005;29:169-75.

37 Daniel OG, Alexandra J, Mushmoom K, et al. Pulmonary embolism and increased levels of D-dimer in patients with coronavirus disease. Emerging Infectious Disease journal 2020;26.

38 Terpos E, Ntanasis-Stathopoulos I, Elalamy I, et al. Hematological findings and complications of COVID-19. Am J Hematol 2020.
39 Zhang L, Yan X, Fan Q, et al. D-Dimer levels on admission to predict in-hospital mortality in patients with Covid-19. J Thromb Haemost 2020;18:1324-9.

40 Zhang W, Zhao Y, Zhang F, et al. The use of anti-inflammatory drugs in the treatment of people with severe coronavirus disease 2019 (COVID-19): the perspectives of clinical immunologists from China. Clin Immunol 2020;214:108393.

41 Chen F, Teachey DT, Pequignot E, et al. Measuring IL-6 and sIL-6R in serum from patients treated with tocilizumab and/or siltuximab following CAR T cell therapy. J Immunol Methods 2016;434:1-8.

42 Du Y, Tu L, Zhu P, et al. Clinical features of 85 fatal cases of COVID-19 from Wuhan. A retrospective observational study. Am J Respir Crit Care Med 2020;201:1372-9.

43 Grasselli G, Zangrillo A, Zanella A, et al. Baseline characteristics and outcomes of 1591 patients infected with SARS-CoV-2 admitted to ICUs of the Lombardy region, Italy. JAMA 2020;323:1574-81.

44 Richardson S, Hirsch JS, Narasimhan M, et al. Presenting characteristics, comorbidities, and outcomes among 5700 patients hospitalized with COVID-19 in the new York City area. JAMA 2020;323:2052.

45 Mehra MR, Desai SS, Ruschitzka F, et al. Retracted: hydroxychloroquine or chloroquine with or without a macrolide for treatment of COVID-19: a multinational registry analysis. The Lancet 2020.

46 , 2020. Available: https://www.recoverytrial.net/news/ statement-from-the-chief-investigators-of-the-randomisedevaluation-of-covid-19-therapy-recovery-trial-onhydroxychloroquine-5-june-2020-no-clinical-benefit-from-use-ofhydroxychloroquine-in-hospitalised-patients-with-covid-19

47 Cao B, Wang Y, Wen D, et al. A trial of Lopinavir-Ritonavir in adults hospitalized with severe Covid-19. N Engl J Med Overseas Ed 2020;382:1787-99.

48 Horby P, Lim WS, Emberson J, et al. Effect of dexamethasone in hospitalized patients with COVID-19: preliminary report. medRxiv 2020.

49 Liu J, Zheng X, Huang Y, et al. Successful use of methylprednisolone for treating severe COVID-19. J Allergy Clin Immunol 2020. doi:10.1016/j.jaci.2020.05.021. [Epub ahead of print: 29 May 2020].

50 Lai C-C, Liu YH, Wang C-Y, et al. Asymptomatic carrier state, acute respiratory disease, and pneumonia due to severe acute respiratory syndrome coronavirus 2 (SARS-CoV-2): facts and myths. J Microbiol Immunol Infect 2020:53:404-12.

51 Felsenstein S, Herbert JA, McNamara PS, et al. COVID-19: immunology and treatment options. Clin Immunol 2020;215:108448.

52 Tay MZ, Poh CM, Rénia L, et al. The trinity of COVID-19: immunity, inflammation and intervention. Nat Rev Immunol 2020;20:363-74.

53 Hunter CA, Jones SA. II-6 as a keystone cytokine in health and disease. Nat Immunol 2015;16:448-57.

54 Pedersen SF, Ho Y-C. SARS-CoV-2: a storm is raging. J Clin Invest 2020;130:2202-5.

55 Tisoncik JR, Korth MJ, Simmons CP, et al. Into the eye of the cytokine storm. Microbiol Mol Biol Rev 2012;76:16-32.

56 Huet T, Beaussier $\mathrm{H}$, Voisin $\mathrm{O}$, et al. Anakinra for severe forms of COVID-19: a cohort study. Lancet Rheumatol 2020;2:e393-400.

57 Eng SS, DeFelice ML. The role and immunobiology of eosinophils in the respiratory system: a comprehensive review. Clin Rev Allergy Immunol 2016;50:140-58.

58 Lindsley AW, Schwartz JT, Rothenberg ME. Eosinophil responses during COVID-19 infections and coronavirus vaccination. J Allergy Clin Immunol 2020;146:1-7.

59 Zhang H, Zhou P, Wei Y, et al. Histopathologic changes and SARSCoV-2 immunostaining in the lung of a patient with COVID-19. Ann Intern Med 2020;172:629-32.

60 Mahallawi WH, Khabour OF, Zhang Q, et al. Mers-Cov infection in humans is associated with a pro-inflammatory Th1 and Th17 cytokine profile. Cytokine 2018;104:8-13.

61 Wu D, Yang XO. Th17 responses in cytokine storm of COVID-19: an emerging target of JAK2 inhibitor fedratinib. J Microbiol Immunol Infect 2020;53:368-70.

62 Spellberg B, Edwards JE. Type 1/type 2 immunity in infectious diseases. Clin Infect Dis 2001;32:76-102.

63 Fu B. Personal communication 2020.

64 Ranney ML, Griffeth V, Jha AK. Critical Supply Shortages - The Need for Ventilators and Personal Protective Equipment during the Covid-19 Pandemic. N Engl J Med 2020;382:e41. 


\section{Correction: Outcomes and biomarker analyses among patients with COVID-19 treated with interleukin 6 (IL-6) receptor antagonist sarilumab at a single institution in Italy}

Montesarchio V, Parrella R, Iommelli C, et al. Outcomes and biomarker analyses among patients with COVID-19 treated with interleukin 6 (IL-6) receptor antagonist sarilumab at a single institution in Italy. J Immunother Cancer 2020;8:e001089. doi: 10.1136/jitc2020-001089.

This paper has been corrected since it was published online. The author name Roberto Parrella was incorrectly spelt as Roberto Parella.

Open access This is an open access article distributed in accordance with the Creative Commons Attribution Non Commercial (CC BY-NC 4.0) license, which permits others to distribute, remix, adapt, build upon this work non-commercially, and license their derivative works on different terms, provided the original work is properly cited, appropriate credit is given, any changes made indicated, and the use is non-commercial. See http://creativecommons.org/licenses/by-nc/4.0/.

(C) Author(s) (or their employer(s)) 2020. Re-use permitted under CC BY-NC. No commercial re-use. See rights and permissions. Published by BMJ.

J Immunother Cancer 2020;8:e001089corr1 . doi:10.1136/jitc-2020-001089corr1

A) Check for updates 